\title{
Erinnerungsnarrative der Vertreibung in der deutschsprachigen Literatur vor und nach der Wende
}

\begin{abstract}
Memory Studies have examined the impact of the historical events of 1989 on (German) memory culture from multiple perspectives since the 1990s. The literary historian must ask whether the transnational opening that pressed the politics of remembrance after 1989 is also reflected in fictional texts of the same period. This contribution is particularly interested in changes in narratives of the expulsion of Germans from Czechoslovakia after World War II as presented in books published by eight German authors before and after the fall of the Iron Curtain: Johanna Anderka, Gerold Effert, Peter Härtling, Bruno Herr, Gudrun Pausewang, Erica Pedretti, Ilse Tielsch, Gustav Wiese. Czech and German character constellations are examined in these texts to reveal a transformation from nationally related homogenization and particularization in the self-determination of both ethnic groups in early works (initially assumed for texts of the previous period) to a more differentiated representations of characters in later texts. The study's main aim is to reveal both constants and changes in these narratives of the expulsion over time, thus offering an overview of narratives typical for each period and their modifications during the given decades.
\end{abstract}

Keywords: Vertreibungsliteratur, Erinnerungsstrategie, Erinnerungsnarrativ, Interkulturalität, deutsch-tschechische Beziehungen

\section{Einleitung}

Der Beitrag setzt sich zum Ziel, deutschsprachige Erzähltexte zu analysieren, die auf die Zwangsaussiedlung der Deutschen aus der Tschechoslowakei fokussieren, um den Perspektivenwechsel in der Erinnerungspraxis in Bezug auf die „Vertreibung“ vor und nach dem Fall des Eisernen Vorhangs anhand dieses Mediums offenzulegen. Diese Zeitspanne wurde ausgewählt, damit die Wirkung des sozialen Rahmens auf die Narrative der „Vertreibung“ in der deutschsprachigen Literatur sichtbar gemacht werden kann.

„Das europäische Jahr“ 1989, in dem die politische Bipolarität zwischen Ost und West zusammengebrochen ist, trug zum Verlust des ursprünglichen Rahmens der Erinnerung an die Vertreibung bei. Das Ende des Kalten Krieges und der 
bipolaren Ordnung schuf eine neue Grundlage nicht nur für den Dialog zwischen der deutschen und der tschechoslowakischen und tschechischen Regierung, sondern auch für den öffentlichen und literarischen Diskurs in den betroffenen Ländern. Die Bedeutung der historischen Ereignisse von 1989 für den Umbruch in Erinnerungsnarrativen wurde in kulturwissenschaftlichen Arbeiten mehrfach thematisiert. Als Aleida Assmann die europäische Erinnerungsgeschichte des 20. Jahrhunderts skizzierte, entwarf sie eine Entwicklungslinie, in welcher der Weg von der starken Gebundenheit der Erinnerungen an nationale Einheiten, verbunden mit dem Bewahren von positiven Selbstbildern derselben und der Instrumentalisierung von Gedächtniskonstruktionen im Kontext des Kalten Krieges, die der Erhaltung des jeweiligen politischen Status quo vor 1989 dienten, bis hin zur „Erosion der nationalen Mythen“ und zum Blick über die nationalen Grenzen hinaus führt, wie dies für die 1990er Jahre festgestellt wird: „Überall in Europa haben sich in den letzten zehn Jahren die Koordinaten der nationalen Geschichten verschoben und komplexeren Darstellungen Platz gemacht.“ (Assmann 2006, 261)

Wolfgang Welsch stellte vor dem Hintergrund westeuropäischer Entwicklungen fest, dass der traditionelle Kulturbegriff „mit seiner Trias von Homogenitätsfiktion, ethnischer Fundierung und äußerer Abgrenzung “ (Welsch 1994, 7) nun irreführend, wenn nicht gefährlich ist:

Die Kulturen - und dabei habe ich zuerst einmal Kulturen westlichen Typs im Auge - weisen heute eine Verfasstheit auf, die den alten Vorstellungen geschlossener und einheitlicher Nationalkulturen nicht mehr entspricht. Sie haben nicht mehr die Form homogener und wohlabgegrenzter Kugeln oder Inseln, sondern sind intern durch eine Pluralisierung möglicher Identitäten gekennzeichnet und weisen extern grenzüberschreitende Konturen auf. (Welsch 1994, 1)

Die mit 1989 verbundene Öffnung in Richtung „Osten“ im Blick behauptet Welsch, dass die „Austauschprozesse zwischen den Kulturen [...] nicht nur das FreundFeind-Schema, sondern auch die Kategorien von Eigenheit und Fremdheit [überborden]“" (Welsch 1994, 4, 11). Während also Aleida Assmann die Grundlage für ihre Feststellung in den erinnerungspolitischen Ereignissen (Anerkennung und Aufarbeitung der Vichy-Kollaboration, Problematisierung der These über Österreich als „erstem Opfer Hitlers“ u.a.) fand, aus denen das Projekt des transnationalen europäischen Gedächtnisses entwickelt werden sollte, ging Wolfgang Welsch in der Ausarbeitung seines Konzepts der Transkulturalität von der Vorstellung der Ausweitung der Verfasstheit westlicher Demokratien aus. Doch wollen beide - sei es im spezifischen erinnerungspolitischen oder allgemeinkulturellen Feld - markante Verschiebungen beobachten.

Aus literaturgeschichtlicher Perspektive ist es von Interesse, ob sich die transnationale Öffnung in der Erinnerungspolitik und die neuen ,transkulturellen Lebensformen“ (Welsch 1994, 11) auch in den literarischen Werken derselben 
Zeitspanne manifestieren. Diese facettenreiche Frage kann man im Rahmen eines begrenzten Beitrags nur unter einem ihrer Aspekte zu beantworten versuchen. So gehe ich in meinem Beitrag zur Darstellung der Zwangsaussiedlung der Deutschen aus der Tschechoslowakei nur auf die deutschsprachige Literatur ein und untersuche darin anhand meines Korpus, inwieweit die Öffnung des Jahres 1989 auf die Narration der Vertreibung in der deutschsprachigen Literatur abfärbt. Auf die Darstellung der Zwangsaussiedlung der Deutschen aus der Tschechoslowakei in der tschechischen Literatur, die sich im transnationalen Rahmen von dem kollektiven Narrativ der „Abschiebung“ zum individualisierten Narrativ der „Vertreibung“ verschiebt, gehen andere Beiträge in diesem Band ein. In den von mir ausgewählten deutschsprachigen Erzähltexten, die in den 1980er und 1990er Jahren auf die Vertreibung fokussieren, wurden dann die deutsch-tschechischen Figurenkonstellationen in den Blick genommen und analysiert, um zu zeigen, inwieweit ein Wechsel von der national bedingten Homogenisierung und Partikularisierung in der Selbstbestimmung beider ethnischen Gruppen hin zur differenzierten, auf diverse Transgressionen fokussierten Darstellung der Figuren in diesen Texten festzustellen ist.

Das Korpus der zu analysierenden Texte wurde auf der Grundlage von thematischer Kontinuität aus Werken von Schriftstellern zusammengestellt, die sowohl in den 1980er als auch in den 1990er Jahren mindestens je einen Text mit Vertreibungsthematik publiziert haben. Dabei bin ich von Axel Dornemanns Bibliografie Flucht und Vertreibung (2005) ausgegangen, von welcher Texte mit der Markierung „Sudetenland“ in die Analyse aufgenommen wurden. Das auf diese Weise entstandene Korpus würde 34 Texte enthalten. Für den vorliegenden Beitrag wurde die Anzahl der Texte weiter reduziert, indem die chronologisch am weitesten voneinander entfernten Werke in Betracht gezogen wurden. So enthält das Korpus sechzehn Texte von acht SchriftstellerInnen (Johanna Anderka, Gerold Effert, Peter Härtling, Bruno Herr, Gudrun Pausewang, Erica Pedretti, Ilse Tielsch, Gustav Wiese). Diese Texte sollen im Folgenden auf ihre Figurenkonstellation hin analysiert werden. ${ }^{1}$

In den Erzählwerken werden zuerst Rollenzuweisungen, Grenzziehungen und Transgressionen bezüglich der dargestellten tschechischen und deutschen

1 Analysen von Figurenkonstellationen in ausgewählten Werken der deutsch- und tschechischsprachigen Literatur wurden bereits von Peroutková (2006) oder Petrbok (2014) durchgeführt. In diesen Publikationen wurden auch die Erinnerungsnarrative exemplarisch dargestellt, durch welche beide Literaturen in den fiktionalen Texten von 1951 bis 2006 gekennzeichnet sind. Im Unterschied dazu ist das Ziel des vorliegenden Aufsatzes, konsequenter auf die Werke aus zwei Jahrzehnten einzugehen, damit die Verschiebungen in den Erinnerungsnarrativen in der ausgewählten Zeitspanne in Bezug auf den Wandel des sozialen Rahmens betrachtet werden können. 
Figuren untersucht, um die Perspektivierung der Erinnerungsnarrative in den jeweiligen Texten zu identifizieren. Berücksichtigt werden hierbei vor allem fiktionale Texte; faktuale Texte werden dagegen nur erwähnt, wenn in ihnen die Verschiebungen in Erinnerungsnarrativen ausgeprägt zum Vorschein kommen. Die verdichteten Konstellationen werden abstrahiert und auf dieser Basis in einem Überblick der vorhandenen Rollenmodelle in Erinnerungstexten der 1980er Jahre „synchron“ gegenübergestellt, wobei auch auf die unterschiedlichen Erzählperspektiven eingegangen wird. Das Hauptziel des Beitrags ist es jedoch, Konstanten und Veränderungen in dem Werk desselben Autors vor und nach 1989, d.h. den diachronen Wandel in der Narration der Vertreibung aufzuzeigen.

\section{Figurenkonstellationen in den Texten der 1980er Jahre: Rollenmodelle des Gegen-, Mit- und Ineinander}

Gustav Wieses Buch Das Amulett aus Böhmen (1983) beinhaltet fünfzehn Geschichten, die nicht nur um Gedichte bereichert sind, sondern denen auch eine „Kurzfassung der Geschichte Böhmens“ im Vorwort vorausgeht und an die eine „Zeittafel zur Geschichte Böhmens“ am Buchende angeschlossen ist, ${ }^{2}$ woraus der Eindruck von Vollständigkeit und Abgeschlossenheit der dargestellten Geschichte des Landes unter dem Aspekt des deutsch-tschechischen Zusammenlebens entsteht. In den historischen Erzählungen wird der nationale Konflikt mit der ständigen Gefährdung der Existenz der deutschen ethnischen Gruppe in den böhmischen Ländern seit dem Jahre 1000 geschildert, deren Kommen und Gehen ins große geschichtliche Kontinuum der geografisch bestimmten Einheit eingeschrieben wird. Im ersten Jahrtausend hätten sich auf diesem Gebiet Bojer, Markomannen und Slawen abgewechselt, die Herzöge der letzteren luden „um das Jahr des Herrn 1000“ (Wiese 1983, 76) die ersten Deutschen ins Land ein, damit die unbewohnten Territorien mithilfe der deutschen Kolonisten bebaut werden und davon profitieren konnten. Ihnen wurden durch ausgeschickte Werber Freiheit, Erb- und Heimatrecht durch einen abgeschlossenen Rechtsver-

2 Auf der Titelseite enthält das Buch gleich drei Gattungszuschreibungen: Es soll sich um eine „Sudetensaga“, einen „historischen Roman“ und „historische Geschichten aus zwei Jahrtausenden“ handeln. Auf die Fragwürdigkeit der Gattungsbestimmung wird im Beitrag nicht eingegangen, da sie nicht das eigentliche Betrachtungsobjekt ist. 
trag versprochen (Wiese 1983, 131). Für Wiese ist es wichtig hervorzuheben, dass die Deutschen sich „an die Abmachungen hielten“ (Wiese 1983, 140), obwohl das gegebene Wort immer wieder - in jeder der folgenden Erzählungen - gebrochen wurde, indem die slawische Bevölkerung Widerstand gegen die Ansiedlung der Deutschen in ihrer Gegend leistete. Die Tschechen werden darin klischeehaft als zornige und rachsüchtige Grobiane geschildert, deren Einstellung gegenüber den Deutschen das Kollektiv repräsentiere: „Ihr bezahlt für eure Vorväter, die einmal in unser Land gekommen sind, vielleicht friedlich und gerufen, doch sicher nicht willkommen; Fremdlinge, die uns übertreffen und beherrschen wollten mit ihrer Sprache!“ (Wiese 1983, 177) Von den Deutschen dagegen entsteht ein Bild der gutwilligen, feinen, vernünftigen, „einfachen Leute“, die nach Gottes Gebot ihre slawischen Brüder lieben, alles vergeben und lediglich mit Gottes Zorn drohen können. ${ }^{3}$ Auf der Ebene der personellen Beziehungen sind einige Beispiele für deutsch-tschechisches friedliches Zusammenleben vorhanden (Liebesgeschichten, Rettung der Deutschen während des Massakers in Ústí nad Labem/Aussig). Dies lässt sich jedoch nicht als ein Versuch deuten, das in jeder Erzählung vorkommende schwarz-weiße Darstellungsmuster gezielt zu relativieren, da der Anteil solcher (positiv besetzten) Figuren an der Gesamtzahl der tschechischen Figuren gering ist, diese Figuren an der Handlungsperipherie situiert sind und nicht vermögen, das Hauptnarrativ über die Deutschen als ewige Opfer der tschechischen Täter zu unterminieren. Die Textstellen mit solcher Thematik stellen eher Ausnahmen dar.

Wieses Argumentation ist vielmehr durch andere, größere Widersprüche gekennzeichnet. In der Erzählung „Ein goldenes Zeitalter?“ findet sich eine Bemerkung eines deutschen Fährmanns über die „Böhmen“, die viel erdulden können, „doch nie vergessen, was sie erdulden mussten“ (Wiese 1983, 167). Da im Text bis dahin und auch danach jedoch von keiner Ungerechtigkeit die Rede ist, die Tschechen von Deutschen angetan wäre, wirkt diese Textstelle zunächst verwirrend, impliziert aber deutlich die Akkumulation des Neids und des Hasses infolge der kleinen sozialen Ungerechtigkeiten sowie seine gewalttätige Entladung, wie dies etwa in einem Herr-Untertan-Verhältnis von Zeit zu Zeit der Fall ist. In „Die einfachen Leute“ kommt weiterhin bei der Beschreibung der Ereignisse der 1930er Jahre die Rhetorik der älteren Generation der Sudetendeutschen Landsmannschaft vor, in der die Schuld für den „Ausgleich von 1938 [= Münchner Abkommen] und die Demütigung von 1939 [= Einmarsch und Protektorat Böhmen und Mähren]“ den Tschechen zugeschrieben wird, die sich 1918 nicht an

3 Vgl. „Gebet des Exulanten“: „Wir wollen sie lieben, / die uns vertrieben / [...] und die triumphieren / einst werden verlieren / die Heimat vor Gott“ (Wiese 1983, 212). 
den Grundsatz der „Toleranz aus dem alten [österreichischen] Vielvölkerstaat“ gehalten hätten (Wiese 1983, 355), was deutlich macht, dass diese Toleranz nur in eine Richtung (von den Deutschen zu den kleinen Völkern im Habsburgerreich) gedacht wird. In derselben Erzählung wird auch gegen die These der Kollektivschuld plädiert, die weder den Deutschen noch den Tschechen gegeben werden soll, obwohl die bisherigen Geschichten ein eindeutig negatives Bild der tschechischen Figuren enthalten, denen eine eventuelle Rechtfertigung für Ungerechtigkeiten gegenüber den Deutschen nicht gegönnt wird. Der Erzähler bedient sich des damals unter Sudetendeutschen verbreiteten Opferdiskurses, indem er an mehreren Stellen hervorhebt, dass die Rolle des „Befreiers“ von der Unterdrückung in der Tschechoslowakei Adolf Hitler nur zufällig zugefallen sei (Wiese 1983, 354). Der tschechische Hass wird in dieser Geschichte erneut festgestellt, die Tschechen hätten den Sudetendeutschen „ganz unbegründet die Schuld an ihrer Lage zugeschoben“, denn „im Sudetenland verstand man diese Maßnahmen am wenigsten“ (Wiese 1983, 355), wobei diese „Maßnahmen“ nicht benannt und damit im Unklaren belassen werden und die Verantwortung dafür bezeichnenderweise nach außen - wohl auf das Deutsche Reich, dessen Teil allerdings das „Sudetenland“ 1938 geworden ist - verlagert wird.

Diese stark monologisierte Redeweise in Wieses Werk, bei der die deutschtschechischen Beziehungen von entsprechend eindeutig positiven und negativen Rollenzuweisungen geprägt werden, ist im Korpus der analysierten Texte einzigartig. Diese Figurenkonstellation lässt sich als Modell des Gegeneinanders mit einem vom Erzähler intendierten asymmetrischen Machtverhältnis bezeichnen. In diesem Modell mit ausgeprägten Grenzziehungen zwischen zwei Volksgruppen, für welche die gegenseitige Interaktion als Kampf bzw. immerwährender Angriff von tschechischer Seite verstanden wird, wäre die gemeinsame Existenz nur unter der Bedingung der gegenseitig respektierten Autonomie möglich gewesen. Dieses Erinnerungsnarrativ mit einem positiven Selbstbild und negativen Fremdbild, welches das Jahrhunderte lange Zusammenleben retrospektiv rekonstruiert und in der Vertreibung gipfeln lässt, bestätigt die These, dass die nationalen Erinnerungsnarrative während des Kalten Krieges auch Erzähltexte über die Vertreibung durchdringen.

Im Unterschied zu diesem polarisierten Rollenmodell werden in Bruno Herrs Roman Nacht über Sudeten (1985) die Figuren differenzierter dargestellt. ${ }^{4}$ Die

4 Der Romaninhalt lässt sich folgendermaßen zusammenfassen. Dargestellt wird das erste Nachkriegsjahr im Leben der Einwohner eines erzgebirgischen Dorfes, das zugleich zum letzten Jahr wird, das der Protagonist Gerhard Neubert in seinem Heimatdorf verbringt. Der sechzehnjährige Gerhard wird im letzten Kriegsmonat eingezogen, zufällig entkommt er der russischen 
stabile Einheit sollen dabei die alteingesessenen deutschen und tschechischen Dorfbewohner bilden. Ihre Beziehungen werden zwar im Buch nicht detailliert dargestellt, der Erzähler deutet jedoch an einigen Stellen an, dass die Alteingesessenen miteinander befreundet waren, sodass einige Tschechen den $\mathrm{zu}$ „evakuierenden“ Deutschen halfen. Dies ist für den Erzähler insofern wichtig, als er mehrmals den demografischen Wandel der tschechischen Volksgruppe hervorhebt, der im ersten Nachkriegsjahr dazu führte, dass die zugewanderten Tschechen („neue Okkupanten“ genannt, Herr 1985, 124) ihre alteingesessenen Landsleute ,in den Hintergrund gedrängt“ haben, und ihnen nun misstrauten. Die Gewalttaten an Deutschen und ihre „Evakuierung“ (Herr 1985, 93) werden von zugewanderten Tschechen und russischen Soldaten verübt, wobei die zugewanderten Tschechen stark abwertend beschrieben werden:

Der Plebs aus dem Inneren Böhmens hatte sich in Bewegung gesetzt; nach Norden und Westen strömten sie, in die seit Jahrhunderten von Deutschen besiedelten Randgebiete, Habenichtse, Glücksritter, die ihr Eldorado suchten und fanden. (Herr 1985, 128)

Dieser Gruppe gehört auch Pavel an, der das Haus des Protagonisten Gerhard Neubert nach der Enteignung übernahm und in den sich Gerhards Schwester Helga am Ende der Erzählung verliebt. Ihre Eheschließung könnte vom Autor als symbolische Versöhnung der antagonistischen Volksgruppen intendiert gewesen sein und für den Neuanfang der deutsch-tschechischen Beziehungen stehen. Es muss jedoch festgestellt werden, dass diese Wende in Helgas Verhalten Pavel gegenüber psychologisch unmotiviert ausfällt und äußerst unplausibel wirkt. Das Bemühen des Erzählers um eine Versöhnung der Protagonisten auf der persönlichen Ebene korrespondiert andererseits mit der Darstellung der alteingesessenen deutschen und tschechischen Dorfbewohner, die jenseits der ethnonationalen Trennlinien normal zusammenleben, worin sich Herrs Roman von Wieses Werk unterscheidet. Deswegen steht Herrs Roman für ein anderes Modell der Figurenkonstellation, das sich als Modell des Miteinander benennen lässt.

Im Unterschied zu Das Amulett aus Böhmen wurden die Ereignisse der deutsch-tschechischen Geschichte in Herrs Roman auf solche Art ausgewählt, dass das Bild einer bis in die 1940er Jahre beinahe konfliktfreien Gemeinschaft entsteht. Die deutschen Dorfbewohner werden mit großer Zuneigung des Erzählers geschildert; auch wenn manche von ihnen Mitglieder der Sudetendeutschen Partei waren und staatliche Macht ausübten, werden sie trotz ihrer Einbindung

Gefangenschaft und kehrt nach Hause zurück, um zum Zeugen der Ereignisse zu werden, die die deutsch-tschechischen Beziehungen im Dorf bestimmen. 
in die Institutionen des NS-Regimes mit dem Argument der Bedeutungslosigkeit des Dorfes von jeder Schuld freigesprochen. Solche Entschärfung führt zur Etablierung eines positiven Bildes der deutschen Dorfbewohner. ${ }^{5}$ Die Auseinandersetzung mit der nationalsozialistischen Vergangenheit sowie die Einbindung der Vertreibungsereignisse in den zeitgeschichtlichen Zusammenhang fehlen in Herrs literarischer Darstellung, weswegen das Erinnerungsnarrativ der ohne Schuld vertriebenen Deutschen in seinem Text deutlich zum Vorschein kommt. Die zugewanderten Tschechen werden folglich dafür angeklagt, dass sie die Unschuldigen bestraften und gegenüber den Tschechen, die diesen gegenüber offen waren oder ihnen halfen, keine Gnade zeigten. Die tschechischen Figuren kommen im Roman nicht selbst zu Wort, ihr dargestelltes brutales Verhalten wird lediglich vom Standpunkt der Deutschen erklärt, mit denen der Protagonist Gespräche führt. Der deutsch-tschechische Konflikt und dessen Lösung werden im Roman trotzdem auf eine ausgeglichenere Weise dargestellt. Das auch in diesem Buch vorhandene Modell des Miteinander wird durch eine pazifistische und versöhnende Erzählintention geprägt. Die Absenz des Willens, der anderen Seite zuzuhören, und die Versuche, die Verwicklungen mit dem NS-Regime zu relativieren, zeigen, dass das Modell des Miteinander seine Grenzen hat und lediglich eine Variation des trennenden nationalen Erinnerungsnarrativs darstellt.

Auf der Grundlage der idealisierten Darstellung ausgewählter Ereignisse wird dieses Modell auch in Gerold Efferts Vertreibungserzählungen aus dem Sammelband Im böhmischen Wind (1985) abgerufen. ${ }^{6}$ Die Mehrheit seiner Erzählungen beschreibt unterschiedlich motivierte Besuche von vertriebenen Deutschen in der Tschechoslowakei 20 und 30 Jahre nach der Vertreibung. Dabei erheben die deutschen Figuren keine materiellen Ansprüche, die tschechischen heißen die alten Nachbarn und deren Kinder willkommen, neue Freundschaften werden über Staatsgrenzen hinweg geschlossen. Die deutsch-tschechische Kommunikation wird weder durch sprachliche Hemmungen erschwert, da sie auf Deutsch geführt werden kann, noch durch Fokussierung des ehemaligen Konflikts, sodass die Vertreibung von beiden Seiten als historische, emotional nicht beladene Gegebenheit wahrgenommen wird. Der Erzähler bemüht sich um diese distanzierte Perspektive auch bei der Schilderung der vergangenen Geschehnisse.

\footnotetext{
5 Dieses wird lediglich an einer Textstelle in Frage gestellt, an der erwähnt wird, dass fast in jedem Haus am Kriegsende das Hitler-Porträt und andere NS-Symbolik mit Bedauern verbrannt wurden.

6 Die Sammlung enthält 18 Erzählungen. Die chronologisch am weitesten zurückreichenden Erzählungen stellen Kindheitserlebnisse der unterschiedlichen Ich- oder Er-Erzähler in den Vorkriegsjahren dar, in denen sich keine sozial-politischen Reflexionen finden.
} 
In den Vertreibungserzählungen wird zuerst der Einsatz einiger Tschechen für Deutsche bei der neuen Regierung thematisiert, erst danach kommen Darstellungen von der Not der deutschen Frauen, Kinder und Alten hinzu, wobei nach den Gründen für diese Not und die Vertreibung selbst nicht gefragt wird. Dies bringt ein generalisierendes Bild von unschuldigen Deutschen und den deren Unglück verursachenden Tschechen hervor, obwohl die Beispiele des Lebens miteinander in den Vordergrund gerückt wurden und die Darstellung der am stärksten konfliktbeladenen Ereignisse der Vor- und Nachkriegszeit vermieden wurde.

Diese Linie, der Autoren in der Darstellung des deutsch-tschechischen Zusammenlebens folgen, gipfelt in den 1980er Jahren in Gudrun Pausewangs Texten über das Leben auf der Rosinkawiese. Keines ihrer drei Bücher ist rein fiktional, weswegen sie nicht direkt in die Analyse aufgenommen wurden. Es ist jedoch erwähnenswert, dass in allen Rosinkawiese-Texten vehement für die Versöhnung der Völker und für den Aufbau einer friedlichen Zukunft plädiert wird. Im Unterschied zu Herrs und Efferts Texten weist Pausewang auf den historischen Zusammenhang zwischen der Vertreibung und der deutschen NS-Politik hin, indem der Leser über den Nationalsozialismus aufgeklärt wird. Im nächsten, 1993 erschienen Roman Pausewangs über das Leben im Adlergebirge schwindet jedoch diese Belehrungsgeste und die pazifistische Aufladung. Auf diese Veränderung soll im zweiten Teil des Beitrags eingegangen werden.

Die oben analysierten Werke von Wiese, Herr, Effert und Pausewang bilden die erste Textgruppe, in der die literarische Bearbeitung der Vertreibungsthematik aus der Perspektive eines meistens nullfokalisierten, heterodiegetischen Erzählers durchgeführt wird, der sowohl auf der figuralen als auch auf der auktorialen Ebene einseitige Aussagen über historisch-politische Ereignisse vorlegt, ohne die Stimme dem Anderen (der tschechischen Seite) zu verleihen. ${ }^{7}$ Im Unterschied dazu sind die Texte aus der zweiten Gruppe, der sich Anderkas, Härtlings, und Pedrettis Werke zuordnen lassen, nicht primär auf die Festhaltung historischer Ereignisse ausgerichtet. Die Texte dieser AutorInnen sind von einer stark introspektiven Sicht der sich erinnernden Erzählfiguren gekennzeichnet, die deutsch-tschechischen Beziehungen werden an wenigen Stellen und nur insofern abgebildet, als sie die Identitätsbildung der Erzählfigur bestimmt haben und deswegen für den Erinnerungsprozess bedeutend sind. Es werden die Bedingungen der Erinnerungsarbeit, die Möglichkeit von Verbalisierung und Verschriftlichung der Erinnerungen sowie der Wahrheitsanspruch des Erinnerten in Frage

7 Für die Beschreibung der Textstruktur werden die erzähltheoretischen Konzepte Gérard Genettes (1998) verwendet, die in der modernen Literaturwissenschaft breite Anerkennung gefunden haben. 
gestellt, wodurch das angebotene historische Erinnerungsnarrativ als subjektiv relativiert wird.

In solchen Texten wird die Grenze zwischen fiktionalem und faktualem Erzählen problematisiert, wie dies bei Peter Härtlings stark autobiografischem Buch Nachgetragene Liebe (1980) der Fall ist, dessen Gattung schwierig zu definieren ist. ${ }^{8}$ Die erzählte Zeit gilt hierin bis auf anachronistische Digressionen über gegenwärtige Gefühle des Erzählers ausschließlich dem Leben seines Vaters, was dem Vorhaben des Erzählers entspricht: Es wird geschrieben, um den Vater zu „entdecken“, „den Abstand zwischen uns, Satz um Satz zu verringern“ (Härtling 1980, 287, 220). Der Gestus des Buchs besteht im diskreten Aufbau der neuen Beziehung in der Erinnerungs- und Umdeutungsarbeit des Sohns seinem Vater gegenüber. Die deutsch-tschechischen Beziehungen werden nicht politisch diskutiert, sondern bleiben auf die personelle Ebene, auf den Familienkreis begrenzt. Dieselbe Familienkonstellation wird dann auch im Roman Große, kleine Schwester (1998) abgerufen und in einen größeren geschichtlichen Horizont eingeschrieben, worauf im zweiten Teil des Beitrags einzugehen ist.

Da der größere historische Kontext in Werken dieser Textgruppe unterdeterminiert erscheint, ist die Rekonstruktion der deutsch-tschechischen Beziehungen in diesen Texten erschwert. Für Johanna Anderkas Kurzprosa Mauersegler (1983), deren Gattungsstatus umstritten ist, ist das auch deswegen kaum möglich, weil in dieser aus der Perspektive eines kleinen Mädchens aufgeschriebenen Kindheitserinnerung die gemeinsame Existenz von zwei ethnischen Gruppen nur einmal angedeutet wird, indem die Ich-Erzählerin sich des „Stimmengewirr[s] - deutsche Worte - tschechische Worte“ entsinnt (Anderka 1983, 98), das in ihrem Miteinander für sie zum Heimatbild unabdingbar dazu gehört.

Unter diese Texte mit autodiegetischen Erzählfiguren, deren Sichtweise durch interne Fokalisierung begrenzt wird, reihen sich auch Erica Pedrettis Texte ein. ${ }^{9}$ Im Roman Die Zertrümmerung von dem Kind Karl beginnt die Erzählerin Erica

8 In der Sekundärliteratur wird der Text als autobiografischer Roman oder autobiografischer Bericht bezeichnet. Weder in der Ausgabe von 1986 noch in Band 7 der Gesammelten Werke (1997) gibt es eine Gattungsangabe nach dem Titel. Mithilfe der von Philippe Lejeune ausgearbeiteten Kriterien für das Vorliegen einer Autobiografie lässt sich feststellen, dass Nachgetragene Liebe von der Definition aus gattungsunbestimmt ist und zwischen einem Roman und einem autobiografischen Roman oszilliert, weil der Autor im Text weder einen autobiografischen noch einen „romanhaften“ Pakt eingeht (Lejeune 1994, 31).

9 Für Erica Pedretti ist zwar in den 1980er Jahren die erste Welle der Erzähltexte mit der Vertreibungsthematik vorbei (Harmloses, bitte 1970, Heiliger Sebastian 1973, Veränderung 1977), dennoch werden diese Bücher immer wieder herausgegeben (1979, 1981), wobei der Roman in der Taschenbuchausgabe 1985 den neuen Titel Die Zertrümmerung von dem Kind Karl erhielt. 
nur zögernd die Lebensgeschichte ihrer Nachbarin Frau Gerster aufzuschreiben, die ihr immer wieder Impulse für das Erinnern an die eigene Vergangenheit gibt, sodass das Werk zwei Erzählperspektiven erhält und zeitlich in drei Schichten differenziert wird (Frau Gersters Leben seit 1918, Geschichte der Ich-Erzählerin seit 1945, die von beiden gelebte Gegenwart in den 1970er Jahren). Obwohl die Ich-Erzählerin ihre Abneigung gegenüber der simplifizierenden Sicherheit in Frau Gersters Weltanschauung betont und dieser ihre eigene verunsicherte mehrschichtige Auffassung der Vergangenheit und der Gegenwart gegenüberstellt, sind die Lebensgeschichten der beiden Frauen durch ein gemeinsames Muster gekennzeichnet. Einerseits ist das Muster anthropologisch (Hineingeborensein in eine Familie, Studienjahre, berufliche Beschäftigung, Heirat, Geburt der Kinder), andererseits wird dieses Lebensmuster mit einem ähnlichen zerstörerischen Effekt durch Kriegsereignisse (im Fall von Frau Gerster handelt es sich um den Ersten Weltkrieg, bei der Ich-Erzählerin um den Zweiten Weltkrieg) sowie durch Auswirkungen der staatlichen Minderheitenpolitik in der Friedenszeit beeinflusst. Die Ich-Erzählerin lässt sich durch zufällige Wörter, Übereinstimmung der Namen und vor allem durch das Erkennen von ähnlichen Erlebnissen in Frau Gersters Leben (z.B. Zwangsausreise am Kriegsende, Hunger während der Kriegsjahre, vom Staat sanktionierte Verfolgung, Liebesgeschichte mit dem künftigen Ehemann, Beantragung der Dokumente aus Italien und der Tschechoslowakei für die Heiratsbewilligung) und ihrer eigenen Lebensgeschichte anfangs immer wieder ablenken, im weiteren Verlauf schreibt sie jedoch diese Erinnerungen umfangreicher auf. Weder die Vertreibungsereignisse noch die deutsch-tschechischen Konstellationen sind prägnant beschrieben. Stellenweise finden sich Erwähnungen des Vaters der Ich-Erzählerin, der aus einem deutschen Lager zurückkehrte und nichts erzählen wollte, zudem aber auch Verweise auf die Angst der Ich-Erzählerin vor den uniformierten Russen, Tschechen und Deutschen (Pedretti 1985, 261, 272, 301). Es wird von mehreren Wechseln der Staatsangehörigkeit der Familienmitglieder berichtet: Ohne den Wohnort zu wechseln, wurden sie ungefragt Österreicher, Tschechoslowaken, Deutsche, staatenlos. Der Onkel der Erzählerin war im Krieg bei der Tschechoslowakischen Legion, trotzdem wurden ihm die tschechoslowakische Staatsbürgerschaft und die Rückkehr verweigert, weil er ethnischer Deutscher war. Erst hier fällt das Wort „Vertreibung“ (Pedretti 1985, 317), sodass die Geschichte auch für uneingeweihte Leser eindeutig wird.

Die aufgelisteten Textstellen lassen nur eine geringe Möglichkeit, die Konstellation der tschechisch-deutschen Figuren zu beschreiben. Der Leser kann rekonstruieren, dass die Familie im Zweiten Weltkrieg antifaschistisch gesinnt war und trotzdem nach dem Kriegsende aus der Tschechoslowakei vertrieben wurde. Wie oben bereits erwähnt, sind für Erica Pedretti nicht die konkreten his- 
torischen Ereignisse von Bedeutung, sondern die Problematik von deren Auswirkungen im Leben der beiden Erzählerinnen im Roman. Auf diese Weise wird das Tabuthema berührt, ohne die Geschichte von einer der Seiten her zu deuten oder revanchistisch der anderen Seite gegenüber zu werden. Neben die Erinnerungen Frau Gersters gestellt, sollen die Erfahrungen der vertriebenen deutschen Familie als ein Beispiel der durch staatliche Machtausübung verunstalteten Lebensgeschichten angesehen werden. Der Konflikt wird unterdeterminiert dargestellt, insofern können auch die persönlichen Beziehungen der vertriebenen Familie zu Tschechen oder anderen Deutschen nicht beschrieben werden.

Die genannten Texte von Härtling, Anderka und Pedretti lassen sich als Manifestationen des Modells des Miteinander zusammenfassen, das funktionierende deutsch-tschechische Zusammenleben wird als unhinterfragter Status quo vor der Vertreibung vorausgesetzt. Im Detail bleiben sie jedoch unbestimmt, denn das Zentrum des Erzählens liegt in der Gegenwart der Figuren. Während bei einem transnationalen Erinnerungsmodell das Hinterfragen der eigenen Argumente und ein gegenseitiges Zuhören der konfligierenden Seiten zu erwarten wären, findet man eine solche Haltung in den analysierten Werken nicht, obwohl die universalisierende pazifistische Geste und die Einbindung der Vertreibungsereignisse in den Horizont der Menschheitsgeschichte in Pedrettis Text Die Zertrümmerung von dem Kind Karl eine Grundlage dafür hätten bilden können. Lediglich in der Beschreibung des bemerkenswerten Erzählers in Nachgetragene Liebe von Härtling, den der Autor an einer Textstelle zwischen drei Brünner Wohnungen von Familienmitgliedern mit unterschiedlichen national-politischen Präferenzen pendeln lässt, könnte man das Aufkeimen des Modells eines Ineinander sehen, für welches Überschreitungen nationaler Grenzen in der Figurenrede und -handlung als eines der Kennzeichen gelten können.

Den Überblick über die in den 1980er Jahren vorhandenen Modelle soll die Beschreibung der Figurenkonstellationen in Ilse Tielschs Roman Die Ahnenpyramide (1980) abschließen. Da die Autorin die historischen Ereignisse in den Mittelpunkt des Erzählens stellt und zugleich den rekonstruktiven Charakter des Narrativs über die erinnerte Vergangenheit hervorhebt, befindet sich der Roman an der Grenze zwischen den zwei bisher dargestellten Textgruppen. Wie Pedrettis Werk wird auch Tielschs Roman durch das Schreiben gegen das Vergessen im Angesicht des bevorstehenden Endes der Zeitzeugengeneration motiviert. Ebenso wird in Tielschs Text die Erinnerungsarbeit der Ich-Erzählerin fokussiert, die den Wahrheitsanspruch des Erinnerten mit diversen Strategien zu relativieren sucht. Die eine Strategie der Relativierung wird als Distanzierung der erwachsenen IchErzählerin von ihren eigenen Kindheitserlebnissen realisiert, von denen in der dritten Person berichtet wird. Die Erzählerin spaltet ihre Erinnerungen aus dem Kindesalter von sich ab und konstituiert für diese eine selbstständige fiktionale 
Figur („das Kind Anni“), die auf der intradiegetischen Erzählebene eingeführt wird. Auf der extradiegetischen Ebene erforscht die Ich-Erzählerin die Geschichte ihrer Familie in Archiven, Bibliotheken sowie in Gesprächen mit den Lebenden. Dabei erhebt sie keinen Anspruch, eine allwissende Instanz zu sein, wie es in Wieses Das Amulett aus Böhmen am deutlichsten der Fall war. ${ }^{10}$ Bei Tielsch begrenzt sich die Erzählerin nur auf die Geschichte ihrer Ahnen und bemüht sich, die Archivfunde von den ausgedachten plausiblen Lebensläufen getrennt zu halten.

Das deutsch-tschechische Zusammenleben wird im Text durch unzählige gemischte Heiraten über Generationen hinweg thematisiert. Die Differenzierung wird zwischen beiden Volksgruppen beibehalten, aber es wird kein hierarchisierendes Verhältnis dargestellt: „Man hatte sie ins Land gerufen, verjagt, man ließ sie zurückkehren, man haßte, bewunderte, duldete sie, man vertrug sich mit ihnen, man lebte mit ihnen, man lernte von ihnen.“ (Tielsch 1980, 34) Als Zeichen des Ineinander der beiden Volksgruppen gelten einerseits Annas Familienstammbaum und die Tatsache, dass sich in ihrer Umgebung viele Deutsche mit tschechischen Namen und Tschechen mit deutschen Namen finden, andererseits der alltägliche Sprachgebrauch von Deutsch und Tschechisch, was noch für die Generation von Annas Großeltern und Eltern von Zeitzeugen bestätigt wird. Auch das „Kind Anni“ ist zweisprachig aufgewachsen.

Tschechisch-deutsche Konflikte setzen erst um das Jahr 1913 ein, und die schrittweise Zunahme der nationalen Spannungen wird gezeigt, wobei die Erzählerin Übergriffe auf beiden Seiten fokussiert, bis mit dem Ersten Weltkrieg ein für beide Seiten zerstörerisches Ereignis kommt: „Wenige Stunden später, am Sonntag, geschah, was beide Seiten in gleicher Weise betraf. Die Lawine war losgetreten, die Katastrophe war nicht mehr aufzuhalten.“ (Tielsch 1980, 167) Die Ursache der gesellschaftlichen Polarisierung setzt auf der politischen Ebene ein, wovon auch

10 Das reflektierte Unwissen über historische Ereignisse kommt bei Tielsch bewusst zum Vorschein, was ihren Text markant von Wiese (1983) unterscheidet. Ihren Umgang mit dem Un-/Wissen kann man am Beispiel der deutschen Kolonisten herausstellen. Wiese schildert die Werbung von Deutschen für die Besiedlung Böhmens in zwei Büchern (Das Amulett aus Böhmen, Lange Reise nach Reichenberg) in beinahe identischen Dialogen. Die Werber aus Böhmen versprachen dabei den Deutschen Freiheit und Erbrecht, brachen aber das Versprochene immer wieder über Jahrhunderte hinweg. Diese Logik mündet bei Wiese (1983) erstens in die eindeutige Gegenüberstellung der beiden Volksgruppen, zweitens in die Erzählerintention, die Hinwendung der Sudetendeutschen zu Hitlers Politik zu rechtfertigen. Bei Tielsch (1980) wird die Einwanderung der Deutschen zwar angesprochen, die Erzählerin gibt jedoch zu, dass sie nicht weiß, woher sie gekommen sind, und formuliert hierbei eine rhetorische Frage: ,Was hat man ihnen damals versprochen?“ (Tielsch 1980, 12) 
die bisher stabilen deutsch-tschechischen Beziehungen im Familienkreis und im nächsten Umfeld betroffen sind. Um nicht zu generalisieren, rekonstruiert die Erzählerin die politische Gesinnung der eigenen Familie. Sie entlarvt ihre eigene durchaus positive Einstellung zu den nationalen deutschen und tschechischen Festzügen, an denen sie sich als Kind nicht beteiligen durfte, da die Eltern sich bemühten, abseits vom Konflikt zu bleiben. In Tielschs Roman wird das Ineinander der beiden Volksgruppen als eine auf diversen Ebenen verflochtene Einheit geschildert. Die Familiengeschichte illustriert die sozialen und sprachlichen Transgressionen in den Lebensläufen der deutschen und tschechischen Figuren im geteilten Raum über vier Jahrhunderte hinweg, sodass die Erzählerin aus ihrer Zeit heraus nicht mehr imstande ist, die genaue Herkunft, Nationalität oder Sprache ihrer Vorfahren festzustellen. Dieses Modell des Ineinander der Figurenkonstellation ist stellenweise bereits in Härtlings Nachgetragene Liebe präsent, aber zeigt sich nur in Tielschs Die Ahnenpyramide vollständig entfaltet.

Zusammenfassend lässt sich festhalten, dass die literarische Produktion der auf Deutsch schreibenden, aus der Tschechoslowakei vertriebenen Autoren in den 1980er Jahren drei Modelle (des Gegen-, Mit- und Ineinander) enthielt. Das letzte Modell kommt zwar eindeutig nur in Tielschs Roman vor, aber es ist bemerkenswert, dass die Grenzüberschreitung zwischen nationalen Erinnerungsnarrativen, die in den erinnerungspolitischen Ereignissen erst nach der Wende 1989 postuliert wird, bereits in fiktionalen Texten der 1980er gewagt wurde.

\section{Konstanten und Veränderungen in den Vertreibungstexten der 1990er Jahre}

In Wieses Roman Ein armer Schlucker (1994) wird die Lebensgeschichte Siegmund Sluschnys in den Blick genommen, der 1900 in Folge einer Affäre der Deutschen Amalia Reckziegel mit dem Tschechen Waclav Sluschny [Václav Slušný = Wenzel Anständig] in Gablonz an der Neiße als unehelicher Sohn geboren wurde und sein ganzes Leben lang Ungerechtigkeiten von beiden Seiten erdulden musste. Wiese bricht dabei mit einem locus communis in der Vertreibungsliteratur, bei dem das friedliche Zusammenleben der Vertreter der beiden Volksgruppen durch deutsch-tschechische Liebesgeschichten, Familiengründung und Geburt von zweisprachigen Kindern gesichert wird. ${ }^{11}$ Einerseits verbindet Siegmunds Eltern kein

11 Gustav Wiese selbst hat dieses Muster bei der Gestaltung der deutsch-tschechischen Konstellationen in der Novelle Das Mädchen aus Rybnice (1983) zu benutzen versucht. 
Liebesgefühl, kurze Zeit nach der vom Dechanten erzwungenen Heirat trennen sich Amalia und Waclav und leben fortan in Nachbardörfern, zwischen denen Siegmund pendelt, nachdem sein Leben in dem einen sowie dem anderen Dorf wegen nationalistischen Hasses unerträglich wird. Andererseits wird auch in diesem Buch Wieses hervorgehoben, dass die Tschechen und die Deutschen durch Neid und Hass geteilt wurden. Der Erzähler klärt auf, dass die Verhältnisse sich vor und nach dem Ersten Weltkrieg nicht geändert haben, nur die Rollen vertauscht worden waren, insofern stets eine der ethnischen Gruppen in ihren Rechten diskriminiert wurde. Der Protagonist spielt dabei die Rolle eines naiven Beobachters, er wird unfreiwillig in Konflikte unterschiedlicher Art hineingezogen, kann aber seine Zugehörigkeit zu beiden Volksgruppen nicht zu einem Vorteil ummünzen. Der Autor greift dabei nach einem literarischen Verfremdungsverfahren, um aus Siegmunds Perspektive, die in Wieses Darstellung zwischen Naivität, Schlauheit und Dummheit oszilliert, die Unfassbarkeit des deutsch-tschechischen Konflikts zu zeigen. Der Autor schöpft aber die Möglichkeiten des Verfahrens nicht vollständig aus; gängige deutsche und tschechische Denkweisen und stereotype Reaktionen auf Argumente der Gegenseite werden nicht entlarvt. Siegmund versteht bloß nicht, warum die beiden Volksgruppen nicht gut miteinander auskommen können. Offensichtlich aus diesem Grund ist der Protagonist hellsichtiger als seine Landsleute, lehnt Hitlers Politik bereits vor dem Beginn des Zweiten Weltkriegs ab und positioniert sich als Pazifist: „Ob Deutscher oder Tscheche, wenn nur der verfluchte Krieg nicht wäre!“ (Wiese 1994, 199) Auf diese Art wird die Rolle Hitlers eindeutig verurteilt, was in Wieses Werk 1983 noch nicht der Fall war, da der Erzähler sich auf Hitlers Rolle als Erlöser der Sudetendeutschen und auf deren Rechtfertigung eingelassen hatte. Außerdem schwindet im neuen Erinnerungsnarrativ die heftige Anklage des tschechischen Hasses gegen die Deutschen, die Beziehungen werden ausgeglichener beschrieben, obwohl auch hier eine kohärente Wiedergabe der Argumentationsweise durch tschechische Figuren fehlt, was wohl einen überdauernden Verständnismangel zeigt. So wagt Wiese in den 1990er Jahren zwar keinen Schritt zur transnationalen Erinnerungsöffnung, bewegt sich aber in den Figurenkonstellationen und in pazifistisch ausgerichteten auktorialen Kommentaren immerhin vom Modell des Gegeneinander zum Modell des Miteinander.

In den Werken der anderen und SchriftstellerInnen aus der für die 1980er Jahre herangezogenen Gruppe (Gerold Effert, Bruno Herr, Gudrun Pausewang), die in ihren Texten primär den historischen Aspekt der deutsch-tschechischen Existenz im gemeinsamen Raum darstellten, ist eine gegensätzliche Tendenz ersichtlich, wie der folgende Überblick ihrer Erinnerungsnarrative zeigen wird.

Efferts früheres Idealisierungsmodell, das in den 1980er Jahren eine Annäherung bedeutete, findet keine Anwendung. Die Fälle von Transgression im deutsch-tschechischen Miteinanderleben kommen in Efferts Sammlung von 
17 Erzählungen unter dem Gesamttitel Damals in Böhmen (1996) nicht vor; anstelle der in den 1970er Jahren neugeknüpften Kontakte auf der persönlichen Ebene wird die Unmöglichkeit der deutschen Integration in der ČSSR geschildert, die im ersten Band nicht angesprochen wurde. Ähnlich wie in Wieses Ein armer Schlucker werden auch in Efferts neuen Erzählungen Prophezeiungen über die vernichtende Rolle Adolf Hitlers für das Schicksal der Deutschen sowie über das Ende der deutschen Geschichte in Böhmen geäußert.

So erinnert sich der Ich-Erzähler in der Erzählung „Der Prophet“ daran, dass er in seiner Kindheit ein Gespräch mit einem armen Greis führte und von ihm hörte, dass sie alle in zwei oder drei Jahren „davongejagt werden, weit fort, in alle Winde“ (Effert 1996, 25). Obwohl dies aus der Kinderperspektive, die einen Verfremdungseffekt in der Erzählung hervorruft, unvorstellbar war (,Wer wird uns davonjagen?“ - Effert 1996, 25), gewinnt das Kind nach diesem Gespräch selbst die Fähigkeit, das Heranrücken des Unglücks zu spüren:

Erste schwere Regentropfen klatschten auf den Plattenweg. Ihr Geräusch verwandelte sich in das Rasseln ferner Panzer, und ich hörte das Getrappel unzähliger Füße; es waren Tausende, Millionen von Menschen, die man aus ihren Häusern trieb, um sie in Güterwagen wegzuschaffen, fort über die Grenze. (Effert 1996, 27)

Die historischen Ereignisse werden nicht zur Begründung herangezogen, was zu einer Vorstellung der Geschichte als einer unberechenbaren, schicksalhaften, mit den Menschen spielenden Kraft führt. Die einzige historische Figur, auf die in der Sammlung hingewiesen wird, ist Adolf Hitler, in dem der blinde Robert den Ursprung des Unglücks der Sudetendeutschen erkennt:

Wir sind einem Vogelfänger auf den Leim gegangen, einem Wahnsinnigen. [...] Wer sät, wird Sturm ernten [...] und die ganz oben, die haben Sturm gesät. Bald wird er sie davonblasen, für immer, sie alle, aber auch Millionen von uns. (Effert 1996, 28)

Ähnlich wie Wiese in der Darstellung von Siegmunds naiver Scharfsinnigkeit bedient sich Effert in der zitierten Erzählung eines anderen locus communis, bei dem ein Blinder die von Anderen nicht gesehene Wahrheit zu erkennen vermag. Die Prophezeiungen eines Alten und die Wahrheitssprüche eines Blinden werden in den Erzählungen des Sammelbandes erfüllt, in dem vier Geschichten über die Vertreibung der Deutschen aus ihren Häusern und ihren Abtransport in Güterwagen folgen.

Der Reihe der Prophetenfiguren gehört auch der Irrsinnige Karl Rotwengel aus Gudrun Pausewangs Roman Die Rotwengel-Saga (1993) an, in dessen Vorhersagen am deutlichsten die These formuliert wird, dass das Schicksal der Deutschen in Böhmen besiegelt wurde. Als Karl Mitte der 1860er Jahre klein war, wurde er im 
Berg verzaubert, seine Seele wurde in den Kosmos weit vom Körper hinausgeworfen, wodurch Karl seine Prophezeiungsfähigkeit erhielt. Er sah die Verwüstung des Adlergebirges und den Abgang der Rotwengel-Familie bereits in seiner Kindheit vorher. Dem steht jedoch die auktoriale Beschreibung der konfliktlosen Nebeneinanderexistenz der Deutschen und Tschechen bis 1918 entgegen, sodass Pausewangs Roman durch einen markanten Widerspruch gekennzeichnet ist. Die tschechischen Figuren sind im Roman peripher, bis 1918 gehören sie ihrem national klar abgegrenzten Raum an. Als die Lage 1918 und 1945 durch das schrittweise Eindringen der Tschechen in den deutschen Raum des Adlergebirges destabilisiert wird, führt dies zum Anfang des Untergangs des friedlichen deutschen Lebens und zur Vertreibung als Katastrophe für die ganze Bevölkerung.

Der Erzählstrategie in Pausewangs faktualem Text Fern von der Rosinkawiese. Die Geschichte einer Flucht (1989), die als eine präzise, auf Zeitzeugnissen basierende und auf einen künftigen Frieden ausgerichtete Rekonstruktion des Erlebten charakterisiert werden kann, wird in den 1990er Jahren die Fiktionalisierung der erlebten Ereignisse gegenübergestellt, die allerdings durch die Hinzufügung der gängigen Geschichten an den Stellen erweitert wird, an denen der Schriftstellerin die eigenen Erlebnisse fehlten. Wie Effert in Damals in Böhmen gibt Pausewang die Bemühung um Versöhnung auf und statuiert die Unfassbarkeit des geschehenen Konflikts und dessen Folgen.

Dieselbe Tendenz von der pazifistischen Aufladung zur generalisierenden Darstellung der tschechischen Volksgruppe ist in Bruno Herrs Roman Weg ohne Wiederkehr (1995) festzustellen. Der Roman lässt sich chronologisch als freie Fortsetzung der Handlung in Nacht über Sudeten verstehen, an dessen Ende der Protagonist im Herbst 1946 nach Deutschland vertrieben wurde und dort seine Geliebte finden wollte. Im Vergleich mit seinem früheren Werk geht der Autor einer differenzierten Darstellung von beiden Volksgruppen nicht mehr nach. ${ }^{12}$ In einem künstlich wirkenden Gespräch, welches in der monologisierenden Form sowie inhaltlich Wieses Erinnerungsnarrativ von 1983 sehr ähnelt, wird Unverständnis für die geschehende Vertreibung geäußert: „Haben wir uns denn nicht immer vertragen mit den Tschechen?“ (Herr 1985, 31) Als Antwort kommt eine in sich widersprüchliche Bewertung der deutsch-tschechischen Beziehungen, die

12 Die Tschechen-Figuren werden eindeutig als „Peiniger“ bezeichnet, die „nach Jahren der schweigend ertragenen Unterdrückung“ zur Macht kamen und die Deutschen loswerden wollen: „Die Tschechen sind unberechenbar, keine Helden, das waren sie noch nie, höchstens Schwejks, die sich auf möglichst bequeme Art durch die Geschichte mogelten, oder blindwütige Fanatiker.“ (Herr 1995, 105) Die Deutschen hingegen werden im Allgemeinen als die Gruppe der guten einfachen armen Leute geschildert, die den wirtschaftlichen Aufschwung des bewohnten Landes sicherten. Ihre nationalsozialistische Vergangenheit wird nicht reflektiert. 
in eine Anklage der Tschechen mündet. Anfangs wird das friedliche Zusammenleben bis zum Ende des Ersten Weltkriegs behauptet, diese Feststellung aber im darauffolgenden Satz unterminiert, indem die Haltung der neuen tschechischen Regierung den Minderheiten gegenüber mit der unterdrückenden habsburgischen Minderheitenpolitik gleichgesetzt wird. Weder der Sprechende noch der Fragende nimmt wahr, dass die zweite Behauptung die Bedeutung der ersten widerlegt: Wenn die tschechische Regierung in der Minderheitenpolitik so versagte wie zuvor die Habsburger, so kann das deutsch-tschechische Zusammenleben auch vor dem Ersten Weltkrieg nicht unproblematisch gewesen sein.

Die beschriebenen Figurenkonstellationen in den neuen Werken von Effert, Herr und Pausewang unterscheiden sich sowohl vom Modell des Gegeneinander, dem die immerwährende Feindschaft der ethnischen Gruppen zugrunde liegt, als auch vom Modell des Miteinander, für das die Versöhnungsgeste sowie das Zugestehen eines friedlichen Miteinanderlebens unter Bedingung der allgemeinen Respektierung der Menschenrechte typisch sind. Daher soll eine andere Kategorie eingeführt werden, um die Figurenkonstellationen zu bezeichnen. Diese sind durch folgende Merkmale charakterisiert: „ein internes Homogenitätsgebot und ein externes Abgrenzungsgebot“ (vgl. Welsch 1994, 4-5, Welsch 2012, 27), ${ }^{13}$ die Auswahl jener historischen Ereignisse, bei der die friedliche Existenz der Kulturen mit klar gezogenen nationalen Grenzen behauptet werden kann, schließlich die Unfassbarkeit vom Ende des Nebeneinanderlebens, die ihrerseits mit dem Unvermögen zusammenhängt, die Argumente der anderen Seite nachzuvollziehen. Solche Konstellationen werden im Folgenden als Modell des Nebeneinander bezeichnet. An den Merkmalen dieses Modells ist ersichtlich, dass es mit einer transnationalen Öffnung im Erinnerungsnarrativ nicht kompatibel ist, was für die Fragestellung des Beitrags von Bedeutung ist, insofern, als für Effert, Herr und Pausewang eine Hinwendung vom versöhnungsorientierten Modell des Miteinander der 1980er Jahre zum separierenden Modell des Nebeneinander festgehalten werden muss.

Die Autoren aus der Textgruppe mit dem Fokus auf eine introspektiv gerichtete Identitätssuche gehen in den 1990er Jahren allmählich zur literarischen Darstellung der geschichtlichen Bedingtheit des Lebens des Einzelnen über. So wird in Pedrettis Roman Engste Heimat (1995) eine aus mehreren Perspektiven zusammengesetzte Reflexion der historisch-politischen Ereignisse entwickelt, wobei das universalisierende pazifistische Narrativ beibehalten wird, in dem gegen die machtpolitischen Regelungen unterschiedlicher Regime argumentiert wird, die

13 Zur kritischen Auseinandersetzung mit Welschs Konzept der Transkulturalität vgl. Heimböckel und Weinberg (2014, 119-144). 
Zwänge auf persönliche Lebensläufe ausüben. Während im ersten Roman keine Grenzziehung auf der persönlichen Ebene thematisiert wurde, werden in Engste Heimat die Beziehungen der Familienmitglieder zu Tschechen sowie zur tschechischen Sprache differenzierter geschildert, wobei man in der Sekundärliteratur widersprüchliche Bewertungen von Pedrettis Darstellung der deutsch-tschechischen Konstellationen in diesem Roman findet. ${ }^{14}$

Die Konstellationen der Romanfiguren werden durch deren soziale, politische, ethnische und sprachliche Positionierungen bestimmt. Anders als in Pedrettis früherem Werk werden Grenzziehungen auch auf der Ebene der Familie realisiert. Bereits die erste Szene von Annas Fremdsprachenunterricht ist bemerkenswert, denn sie zeigt ihre Abstammung aus einer bürgerlichen, finanziell gut versorgten Familie, deutet aber andererseits eine Isolation der Familie von der Umgebung an: Anna bekommt Englisch- und Französisch-Unterricht, kann jedoch nicht die zweite Sprache ihres Heimatlandes sprechen. Während die Eltern Tschechisch „abstoßend fanden, so daß sie den Kindern jede Annäherung, den kleinsten Akzent abzugewöhnen versuchten“ (Pedretti 1995, 34), erlernt Anna in Gesprächen mit dem tschechischen Mädchen Jarmila einige Wörter. Auch dreißig Jahre nach der Vertreibung bedeutet für Anna die Sympathie für das Tschechische eine „Abwendung von der Familie“ (Pedretti 1995, 34).

Die Familie bleibt lange von den Kriegsereignissen hinter der Gartenmauer verschont, die die eigentliche Grenze von Annas Kindheitswelt bildete und nach

14 So setzt sich etwa Henriette Herwig mit den Thesen Valentina Glajars auseinander, die Erica Pedretti unterstellt, einen revisionistischen Text geschrieben zu haben, da im Roman die Mehrheitsunterstützung der Sudetendeutschen Partei durch die deutsche Bevölkerung in der Ersten Tschechoslowakischen Republik sowie deren Beteiligung an den NS-Verbrechen im Protektorat Böhmen und Mähren nicht reflektiert würden. Herwig betont, dass Pedretti diese Umstände nicht verschweige, sondern diese bloß im Roman nicht Vordergrund stünden (Herwig 2012, 211). Man könnte jedoch die Äußerung der beiden Literaturwissenschaftlerinnen relativieren und in Zusammenhang mit ihren eigenen Kontexten stellen. Henriette Herwig, die den Lehrstuhl für Germanistik an der Universität Düsseldorf bekleidet, gehört zu den „eingeweihten“ Lesern und kann daher explizite Erwähnungen von bestimmten historischen Fakten in Pedrettis Buch entbehren. Die im Text vorhandenen Andeutungen können diesem Lesertyp ausreichen, um den Kontext zu rekonstruieren. Valentina Glajar, die sich an Pedrettis „unterdeterminierter“ Erzählweise stößt, rechnet dagegen mit „uneingeweihten Lesern“ (,uninitiated readers“), die die Einzigartigkeit von Pedrettis Protagonisten Gregor, der sich in die antifaschistisch gesinnte Minderheit der Sudetendeutschen einreiht, ohne Kenntnisse von unerwähnten historischen Ereignissen nicht bemessen können (vgl. Glajar 2004, 94). Daher müsse Pedrettis Roman mit den historischen und politischen Gegebenheiten der Ersten Tschechoslowakischen Republik konfrontiert werden, um für die Leser eine ausbalancierte Darstellung von den zur Vertreibung führenden Geschehnissen zu erzielen (vgl. Glajar 2004, 104). 
dem Kriegsende zerstört wurde. Valentina Glajar schlägt vor, diesen Eskapismus als eine Reaktion auf die Marginalisierung der Deutschen im neuen tschechoslowakischen Staat anzusehen (vgl. Glajar 2004, 84). Als die politischen Ereignisse diesen Separatismus unmöglich machten, wurde eine andere Grenze zwischen der Familie und der Umgebung gezogen: Annas Onkel musste fliehen, ihr Vater geriet in ein deutsches Lager, da sich die beiden offen gegen Hitlers Politik äußerten. Für Pedretti wird diese Konfliktverschiebung vom Nationalen zum Politischen zu einem Prüfstein für die Bewertung jeder Romanfigur. Sie betont, dass manche Deutschen und Tschechen durch einen nationalistischen Hass geteilt werden, dieser Hass wurde von einer Generation zur anderen weitergegeben, explodierte auf beiden Seiten; er war in den 1970er Jahren immer noch präsent und richtete sich um 1990 neben den Deutschen auch gegen Roma. Daneben gab es schon immer Tschechen, die persönliche Beziehungen zu den Deutschen pflegten und hilfsbereit waren. In Annas Familie findet man beide Einstellungen, die in den Jahren nach der Vertreibung die unterschiedlichen Einstellungen zum Tschechischen widerspiegeln. Der Vater verbietet Anna, in die Tschechoslowakei, ,an diesen Ort der Enttäuschung, der Schmerzen“, zu reisen (Pedretti 1995, 54, 176, 178), sie riskiert dies aber und will wie Gregor aus der konfliktbeladenen Kontinuität aussteigen. Die verunsicherte, sich das ganze Leben lang um Verständigung bemühende Figur wird nur „erleichtert“, wenn sie alles hinter sich lässt, und kann erst im Exil ein Sicherheitsgefühl verspüren (Pedretti 1985, 272). Wie im ersten analysierten Roman manifestiert sich auch in diesem Text Pedrettis bei der Figurenkonstellation das Modell des Miteinander.

Pedrettis Erzählstrategie, bei der die Vertreibung mit anderen historischen Beispielen von staatlicher Diskriminierung und Verfolgung aus nationalen Gründen parallelisiert wird, findet man auch in Ilse Tielschs Buch Eine Winterreise (1999). Letzteres ist nicht direkt der Vertreibungsthematik gewidmet und reiht sich nicht in die fiktionalen Texte ein. Während die Autorin durch das Bulgarien der 1990er Jahre reist, stellen sich ihre Erinnerungen an die eigene Kindheit in Südmähren ein, weil sie „manches in der Geschichte dieses Landes“ an gesellschaftlich-politische Zustände erinnert, die sie im Kindesalter erlebt hat (Tielsch 1999, 27). Gemeint sind hiermit vor allem die Bevorzugung und Unterdrückung von Sprachen und Religionen: In beiden Fällen wurde die slawische Kultur einer anderen untergeordnet. In Bezug auf die Kindheit werden die deutsch-tschechischen Konstellationen - wie in Tielsch (1980) - dennoch durch Gebrauch beider Sprachen im Alltag sowie durch Eheschließungen dargestellt:

Und wahrscheinlich hat es, wie in der Welt des Kindes, das ich gewesen bin, Menschen gegeben, die aus den verschiedenen Sprachgruppen stammten und in Liebe miteinander verbunden waren, die das Wagnis einer Ehe miteinander eingingen und Kinder zeugten, [...] 
welche [...] gleichmäßig beeinflusst von Vater und Mutter, gemischt aus den Farben beider Nationen und beheimatet in beiden Sprachen, ein anderes Bewußtsein [...] entwickelten als Töchter und Söhne aus Familien, in denen nur eine Sprache gesprochen, nur eine Tradition gepflegt wurde. (Tielsch 1999, 28)

Die Autorin schlussfolgert, dass solche Kinder von den Vertreibungen am schwersten getroffen werden, aber auch ,eine der wenigen wirklichen Hoffnungen unserer Welt“ sind, denn ihnen wird in ihren Familien beigebracht, dass „,nur menschliche Qualitäten und menschliche Bindung zu zählen“ haben (Tielsch 1999, 29). Solche humanistischen Passagen bestimmen den Reisebericht, in dem Geschichten der vielfältigen Unterdrückungskonstellationen parallelisiert werden: Bulgaren unterdrückt von Griechen, Türken, Deutschen und Russen, Tschechen von Deutschen, alteingesessene Deutsche nach dem Zweiten Weltkrieg von Tschechen und Bulgaren. Im deutsch-tschechischen Konflikt werden keine Schuldigen ausgemacht, die Autorin plädiert für Unterlassen von Denunzierungen und für Toleranz dem Anderen gegenüber. Das Erinnerungsnarrativ bleibt dasselbe wie im Roman Die Ahnenpyramide (1980), während die Figuren der Deutschen und Tschechen im neuen Text angesichts dessen faktualen Charakters eines Reiseberichtes nicht näher bestimmt werden können.

Die Schwierigkeiten in der Bestimmung der Figurenkonstellation betreffen auch Johanna Anderkas neue stark autobiografische Textsammlung Bewahrte Landschaft (1999). Darin stellt sie die Unmöglichkeit, sich an die Landschaft mit Namen „von Orten und Flüssen und Bergen“ zu erinnern, fest und will „das innere Land“ (Anderka 1999, 8-9) verschriftlichen, das im Laufe der Zeit durch Vergessen, Träumen, Angstgefühle, Erinnerungsversuche an das Gehörte und Phantasierte geprägt worden ist. Wie in Tielschs Roman Die Ahnenpyramide und in Pedrettis Werk Engste Heimat trennt sich die Erzählerin von ihrer Kindheit durch die Einführung der Kindesfigur, der die brüchigen Erinnerungssplitter zugeschrieben werden. Demgegenüber stehen die Erinnerungen der Erzählerin an ihr Herkunftsland, die in die unmittelbare Vergangenheit zurückreichen und sich auf die letzte Reise nach Tovačov beziehen. Diese Erinnerungen werden auf der extradiegetischen Ebene in das verallgemeinernde Narrativ über die mitteleuropäische Geschichte hineingenommen. Analog zu Pedrettis Die Zertrümmerung von dem Kind Karl und Tielschs Eine Winterreise wird von dem nationalen Erinnerungskonflikt abgesehen und der ständige Rollenwechsel von Siegern und Besiegten angesprochen: „So viele Kriege. Übereinandergeschichtet ihre Spuren. Andere Sieger und andere Besiegte und doch die gleiche Angst, die gleiche Hoffnung und Verzweiflung und der Tod [...].“ (Anderka 1999, 16) Man muss folglich feststellen, dass die Figurenkonstellation bei Anderka sowohl in den 1980er als auch in den 1990er Jahren unterdeterminiert erscheint, weswegen sich anhand 
von ihren Texten kein eindeutiges Modell des deutsch-tschechischen Zusammenlebens rekonstruieren lässt, obwohl manche auktorialen Kommentare das Modell des Miteinander implizieren.

Im Unterschied zu Ilse Tielschs und Johanna Anderkas Werken aus den 1990er Jahren bewegt sich Peter Härtling in die Gegenrichtung: von einer hybriden Gattungsform mit starken autobiografischen Zügen in Nachgetragene Liebe zu dem eindeutig fiktionalen Text Große, kleine Schwester (1998). Im Roman wird die Geschichte Ruths und Leas auf zwei Zeitebenen betrachtet: Die Darstellung ihrer letzten Lebensjahre in der westdeutschen Stadt N. rahmt die intradiegetische Schilderung der Kindheit und Jugend beider Schwestern in ihrem gemischten deutsch-tschechisch-jüdischen Umfeld in Brno/Brünn. Obwohl diese Umgebung in der Elterngeneration durch soziale Unterschiede gekennzeichnet ist (die tschechischen Figuren Zdenka und Pan Lersch sind Bedienstete der deutschen Familie Böhmer und der jüdischen Familie Ribasch), bilden alle Figuren am Romananfang eine gesellschaftlich perfekt funktionierende Einheit, die allmählich durch die Auswirkung von staatlicher nationalistischer Politik zerfällt. Der Erzähler markiert die ersten Verstöße gegen das Weltbild der Schwestern.

Sie gerieten immer tiefer in die Geschichten der Erwachsenen. [...] Niemand überraschte die Schwestern so wie Sarah und Mizzi Ribasch, die sich plötzlich zu den Juden zählten und [...] taten, als sei es schon immer der Fall gewesen, was Herr Ribasch von neuem mit der Erklärung durcheinander brachte, daß er deutsch spreche und denke, als Jude lebe und gewiß tschechischer Staatsbürger werde. Wurde ihnen das Gerede zu viel, drohten sie am Unverstandenen zu ersticken [...]. (Härtling 1998, 62)

Anders als Tielsch in Die Ahnenpyramide, die die Geschichte von vielen Familiengenerationen verfolgt, verdichtet Härtling das Schicksal der letzten Generation der Deutschen vor der Vertreibung in Figuren, deren Sozialisierung in die Entstehungszeit der Ersten Tschechoslowakischen Republik fiel und die sich mit diesem Staat identifizierten, beide Sprachen beherrschten sowie sich als vollberechtigte tschechische Bürger fühlten. In dieser Generation wurde die frühere soziale Asymmetrie zwischen Deutschen und Tschechen aufgehoben, tschechisch-deutsche und tschechisch-jüdische Mischehen wurden geschlossen.

Die tschechisch-deutschen Mischehen werden jedoch im Text als potentiell konfliktbeladen dargestellt, obwohl sich die Figuren um Verständnis bemühen. Das wird an der Textstelle deutlich, an der Lea von ihrem Mann Jiři Pospischil [Jiří Pospíšil] ,in einer Anwandlung von Chauvinismus vorgeworfen [wurde], sie könne seine Trauer um Masaryks Tod als Deutsche nicht teilen“ (Härtling 1998, 181, Hervorhebung von E.M.). Der Erzähler stellt dem „Teilen“ das „Verstehen“ als eine andere Verständigungsmöglichkeit gegenüber. Obwohl Lea als Deutsche das tschechische Erinnerungsnarrativ mit ihrem Mann nicht aktiv zu teilen vermag, 
kann sie es doch verstehen. Es wird die Unmöglichkeit der völligen Aneignung der kollektiven Identitäten des Anderen thematisiert, denn die Gegenseiten werden durch unterschiedliche Sozialisationsmuster geprägt. Lea ist in einer deutschen Familie aufgewachsen und kann folglich - trotz aller Neigung zu den Tschechen - die tschechische Geschichte und Masaryks Rolle nur vom Standpunkt einer Deutschen aus nachvollziehen. Nichtsdestoweniger betrifft diese Differenz nur die politische Ebene, während in Leas Rolle einer „Botin“, die zwischen deutschen und tschechischen Familienteilen pendelt, und in Ruths eklektischen Ansichten und Verhaltensweisen Beispiele für ein Ineinander beider ethnischer Gruppen geschaffen werden. Außerdem zeichnet sich die einzigartige Stellung von Härtlings Roman im analysierten Textkorpus dadurch aus, dass im Text zum ersten Mal die tschechische (wenn auch in gewisser Hinsicht abgemilderte) Sicht des nationalen Konflikts thematisiert wird. Aus diesen Gründen kann das Erinnerungsnarrativ im Roman grenzüberschreitend, transnational genannt werden.

\section{Zusammenfassung}

Der Vergleich der deutsch-tschechischen Figurenkonstellationen in je acht Erinnerungstexten vor und nach der Wende zeigt keine radikale Perspektivenverschiebung in der literarischen Erinnerungspraxis. Im am häufigsten vertretenen Modell des Miteinander von Figurenkonstellationen (Herr 1985, Effert 1985, Härtling 1980, Pedretti 1985, Pedretti 1995, Anderka 1999) werden die deutsche und die tschechische Kultur in sich homogen und mit eindeutig gegebenen Grenzen dargestellt. In den erzählten Geschichten wird das Miteinanderleben in Überschreitungen dieser Grenzen innerhalb der Figurenkonstellationen zugespitzt, was als eine Art Suche „nach Chancen der Toleranz, der Verständigung, der Akzeptanz und der Konfliktvermeidung oder der Konflikttherapie“ (Földes 2009, 516) interpretiert werden könnte. Die Verständigungsmöglichkeit wird in diesen Werken aus der Perspektive des allgemeinen Menschenrechts auf Leben und Freiheit thematisiert, aus der folgt, dass denjenigen, deren Rechte verletzt worden sind, Mitleid entgegengebracht werden soll und man ihrer gedenken solle. Dies wird auf der Ebene von konkreten persönlichen deutsch-tschechischen Beziehungen thematisiert und in beiden Romanen Pedrettis sowie in Tielschs und Anderkas Werken aus den 1990er Jahren durch die Parallelisierung der Vertreibung der Deutschen mit anderen historischen Beispielen von Unterdrückung als inhärentes Problem der Menschheitsgeschichte dargestellt.

Die eingangs angenommene Auswirkung des veränderten sozialen Rahmens auf die Erinnerungsnarrative literarischer Werke, in denen eine transnationale Öffnung des Erinnerns an die Vertreibung sowie an die deutsch-tschechischen 
Beziehungen vermutet wurde, lässt sich in den Texten aus den 1990er Jahren nicht feststellen. Nur in beiden Werken Tielschs und in Härtlings Große, kleine Schwester kommt das Modell des Ineinander der gelungenen sprachlichen und gesellschaftlichen Integration vor, in dem das Fremde und das Eigene nicht auf der nationalen Ebene konstituiert werden. Das gut funktionierende Alltagsleben der Familien, Freundes- und Kundenkreise der deutschen und tschechischen Figuren wird durch politische Polarisierung gestört, die erst im nächsten Schritt die nationalistischen Mechanismen aktiviert. Die Verschiebung vom Modell des Miteinander zum Modell des Ineinander findet sich nur in Härtlings Große, kleine Schwester, obwohl - wie oben angedeutet - diese Konstellation bereits in seinem Werk in den 1980er Jahren im Kern vorhanden ist und im neueren Roman lediglich weiterentwickelt wird.

Abgesehen von diesen Texten hat die Analyse gezeigt, dass es in den 1990er Jahren nicht nur bei der vorherrschenden nationalen Perspektivierung auf der figuralen Ebene geblieben ist, sondern sich auf der auktorialen Textebene eine Bewegung hin zu den nationalen Narrativen zeigt. Davon zeugt beispielsweise der Schwund von pazifistisch gesinnten, versöhnenden Passagen in Pausewangs, Herrs und Efferts Texten der 1990er Jahre. Während solche Kommentare in den Werken derselben Autoren in den 1980er Jahren polarisierten Figurenkonstellationen entgegenstanden, führt ihr Weglassen zur Konstituierung des Modells des Nebeneinander, das in ihren Werken früher nicht präsent war. Bei dieser Konstellation setzen sich die Kulturen voneinander ab, ihr friedliches Leben ist nur bei der Bewahrung der undurchlässigen Grenzen möglich, denn jede Durchdringung des Fremden würde zur Destabilisierung führen. Ein anderes gemeinsames Merkmal dieser Texte ist die Einführung von prophezeienden Figuren. Eine solche Figur hat einen Abstand zum sozialen und politischen Leben (sie ist blind, irrsinnig, naiv, zu alt oder zu jung) und kann deswegen manches scharfsinniger erkennen und künftige Ereignisse vorhersagen. Diese Figuren kommen in Erinnerungsnarrativen vor, in denen es an Verständnis für die Konfliktursachen sowie für die Argumentationsweise der tschechischen Seite mangelt. Die Vertreibung wird darin als eine von der menschlichen Natur herrührende Naturkatastrophe wahrgenommen, die außerhalb des historischen Ursache-Wirkung-Verhältnisses steht.

Es lässt sich feststellen, dass man weder die in den 1990er Jahren gefeierte transnationale Öffnung in der Erinnerungspolitik noch die neue, transnationale Verfasstheit der Kultur in dem Vergleich von Figurenkonstellationen in den literarischen Werken nachweisen kann. Dieses negative Ergebnis geht diachron in beide Richtungen: Einerseits war das Modell des Ineinander bereits in den 1980er Jahren vorhanden, sodass sich von keiner Neuerung der 1990er Jahre sprechen lässt, andererseits spiegelt die häufige Verschiebung vom Modell des Miteinan- 
der zum Nebeneinander in drei von acht analysierten Texten die Tendenz zur nationalen Partikularisierung wider. Es zeigt sich, dass die erwartete Ablösung der nationalen Erinnerungsnarrative durch ein integrierendes transnationales Erinnerungsnarrativ bei der Darstellung der Vertreibung in der deutschsprachigen Literatur im untersuchten Zeitraum kaum vorliegt.

\section{Literaturverzeichnis}

\section{Primärliteratur}

Anderka, Johanna. „Mauersegler“. Heimat Beskidenland. Autoren aus dem Beskidenland erzählen. Hg. Gertrud Hanke-Maiwald. Nürnberg: Helmut Preußler, 1983. 97-99.

Anderka, Johanna. Bewahrte Landschaft. Fulda: Verlag freier Autoren, 1999.

Effert, Gerold. Im böhmischen Wind. Husum: Husum Druck- und Verlagsgesellschaft, 1985.

Effert, Gerold. Damals in Böhmen. Husum: Husum, 1996.

Härtling, Peter. Nachgetragene Liebe. Frankfurt am Main: Luchterhand, [1980] 1988.

Härtling, Peter. Große, kleine Schwester. Köln: Kiepenheuer \& Witsch, 1988.

Herr, Bruno. Nacht über Sudeten. Husum: Husum Druck- und Verlagsgesellschaft, 1985.

Herr, Bruno. Weg ohne Wiederkehr. Freiburg i. Br.: Freiburger Echo Verlag, 1995.

Pausewang, Gudrun. Fern von der Rosinkawiese. Die Geschichte einer Flucht. Ravensburg: Otto Maier, 1989.

Pausewang, Gudrun. Die Rotwengel-Saga. Frankfurt am Main: Eichborn, 1993.

Pedretti, Erica. Die Zertrümmerung von dem Kind Karl. Frankfurt am Main: Suhrkamp, 1985.

Pedretti, Erica. Engste Heimat. Frankfurt am Main: Suhrkamp, 1995.

Tielsch, Ilse. Die Ahnenpyramide. Graz: Styria, 1980.

Tielsch, Ilse. Eine Winterreise. St. Pölten: Literaturedition Niederösterreich, 1999.

Wiese, Gustav. Das Amulett aus Böhmen. Linsengericht, Eidengesäß: G. Wiese, 1983.

Wiese, Gustav. Ein armer Schlucker. Freiburg i. Br.: Freiburger Echo Verlag, 1994.

\section{Sekundärliteratur}

Assmann, Aleida. Der lange Schatten der Vergangenheit. Erinnerungskultur und Geschichtspolitik. München: C.H. Beck, 2006.

Dornemann, Axel. Flucht und Vertreibung aus den ehemaligen deutschen Ostgebieten in Prosaliteratur und Erlebnisbericht seit 1945. Eine annotierte Bibliographie. Stuttgart: Anton Hiersemann, 2005.

Földes, Csaba. „Black Box ,Interkulturalität'. Die unbekannte Bekannte (nicht nur) für Deutsch als Fremd-/Zweitsprache. Rückblick, Kontexte und Ausblick“. Wirkendes Wort 59.3 (2009): 503-525.

Genette, Gérard. Die Erzählung. München: Fink Verlag, 1998.

Glajar, Valentina. The German Legacy in East Central Europe as Recorded in Recent GermanLanguage Literature. New York: Camden House, 2004. 
Heimböckel, Dieter, und Manfred Weinberg. „Interkulturalität als Projekt““. Zeitschrift für interkulturelle Germanistik 5.2 (2014): 119-144.

Herwig, Henriette. „Heimatverlust, Altern und Erinnerung in Erica Pedrettis Spätwerk“. Die Erinnerungstexte der Autorin Erica Pedretti. Hg. Meike Penkwitt. Würzburg: Königshausen \& Neumann, 2012. 209-220.

Lejeune, Philippe. Der autobiographische Pakt. Frankfurt am Main: Suhrkamp, 1994.

Peroutková, Michaela. Literarische und mündliche Erzählungen über die Vertreibung: Ein deutsch-tschechischer Vergleich. Duisburg: WiKu-Verlag, 2006.

Petrbok, Václav. „Obraz a vzpomínka. Několik poznámek k odsunu/transferu/vyhnání sudetských Němců v české a německojazyčné beletrii“. Uzel na kapesníku. Vzpomínka a narativní konstrukce dějin. Hgg. Martina Poliaková, Jakub Raška, Václav Smyčka. Praha: Filozofická fakulta Univerzity Karlovy, 2014. 130-152.

Welsch, Wolfgang. „Transkulturalität - die veränderte Verfassung heutiger Kulturen“. VIA REGIA - Blätter für internationale kulturelle Kommunikation 20 (1994). https://www.via-regia. org/bibliothek/pdf/heft20/welsch_transkulti.pdf (eingesehen am 29.03.2019).

Welsch, Wolfgang. „Was ist eigentlich Transkulturalität?“ Kulturen in Bewegung. Beiträge zur Theorie und Praxis der Transkulturalität. Hgg. Dorothee Kimmich, Schamma Schahadat. Bielefeld: transcript, 2012. 25-40. 